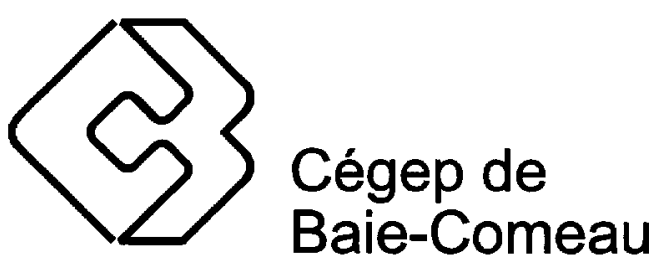

\title{
POLITIQUE ENVIRONNEMENTALE
}





\section{DÉFINITIONS}

\section{Protection de l'environnement :}

La mise en œuvre de moyens destinés à prévenir, diminuer ou éliminer toute affectation nocive, ne serait-ce que partielle, de l'ensemble des conditions naturelles (biologiques, physiques et géographiques) qui agissent sur les organismes vivants, tels que les plantes, les animaux et les humains.

\section{Développement durable :}

«Développement qui répond aux besoins du présent sans compromettre la capacité des générations futures de répondre aux leurs ». II s'agit de la définition officielle rédigée en 1987 par la Commission Brundtland. Elle est reconnue par les Nations Unies et par quelque 180 États et gouvernements. Le développement durable tient compte des trois éléments fondamentaux que sont l'environnement, l'économie et la société ainsi que des relations qu'ils établissent entre eux.

\section{ARTICLE 1- ÉNONCÉ DE PRINCIPE}

Le Cégep de Baie-Comeau, conscient de l'impératif de protéger l'environnement tant pour la génération actuelle que pour celles qui la suivront, affirme son désir d'adopter, de maintenir et de faire respecter des normes sévères en matière de protection de l'environnement et de développement durable.

\section{ARTICLE 2 - PORTÉE DE LA POLITIQUE}

Dans le respect des lois, des règlements ainsi que dans les limites des disponibilités budgétaires du cégep, la présente politique guide ses interventions en matière de :

- Réduction, réutilisation, recyclage et valorisation (3RV)

- Économie d'énergie

- Qualité de l'air

- Gestion de l'eau

- Gestion des déchets

- Gestion des matières dangereuses

- Approvisionnement
- Entretien ménager

- Aménagement extérieur

- Éducation environnementale

- Élaboration, révision et développement de programmes d'études

\section{ARTICLE 3 - DESTINATAIRES}

Cette politique environnementale concerne tous les étudiantes et étudiants et l'ensemble du personnel. Elle s'adresse aussi à toute personne, organisme ou entreprise qui transige avec le Cégep soit comme partenaire, fournisseur ou encore comme simple visiteur. Le Cégep s'attend donc à ce que tout le monde qui se retrouve sur ses propriétés, pour quelque raison que ce soit, se comporte en citoyen responsable face au respect de l'environnement.

\section{ARTICLE 4 - RÔLES ET RESPONSABILITÉS}

La Direction générale se voit confiée l'entière responsabilité de la politique environnementale. En ce sens, elle assume les fonctions relatives à son application et à sa révision. Cependant, elle confie :

- À la Direction des études, les rôles d'éducation environnementale et d'élaboration, de révision et de développement de programmes d'études fidèles au contenu de la présente politique.

- À la Direction des services administratifs, les rôles de récupération, de réutilisation, de recyclage et de valorisation ainsi que ceux d'économie d'énergie, de gardien de la qualité de l'air, de gestionnaire de l'eau, des déchets et des matières dangereuses. De plus, l'entretien ménager, l'aménagement extérieur et l'approvisionnement font aussi partie de son rôle.

- Au comité d'action et de concertation en environnement, le rôle de vigie en matière de protection de l'environnement au cégep et lui confie le mandat de proposer toute amélioration qu'il jugera utile dans le but de faire progresser la cause environnementale dans le milieu. 


\section{ARTICLE 5 - COMITÉ D'ACTION ET DE CONCERTATION EN ENVIRONNEMENT}

La composition du comité est la suivante : deux membres du personnel par catégorie (cadre, professionnel, enseignant, soutien) et auxquels s'ajouteront deux étudiantes ou étudiants. Son rôle est celui indiqué à l'article 4 de la présente politique.

\section{ARTICLE 6 - BUT}

Être reconnu comme citoyen corporatif responsable soucieux de l'environnement.

\section{ARTICLE 7 - OBJECTIFS}

\subsection{Objectif général}

Faire comprendre à toute la communauté collégiale qu'il est vital de se préoccuper d'environnement.

\subsection{Objectif éducatif}

Rendre chaque personne (personnel, étudiantes, étudiants et fournisseurs) plus responsable face aux conséquences qu'ont ses propres choix de consommation sur la détérioration ou la préservation de l'environnement ainsi que sur le développement durable.

\section{ARTICLE 8 - OBJECTIFS SPÉCIFIQUES}

\subsection{Formation}

8.1.1 Avant la fin de l'année scolaire 20082009, former tout membre du personnel concerné par la protection de l'environnement et le développement durable dans l'exercice de ses fonctions ou encore le soutenir s'il désire se perfectionner dans ces domaines.

8.1.2 D'ici la fin de l'année scolaire 2011, intégrer des notions relatives à la protection de l'environnement et au développement durable dans les activités professionnelles du personnel.

8.1.3 À chaque fois que le Cégep révise l'un de ses programmes d'études, intégrer des compétences en matière de protection de l'environnement et de développement durable. Ceci se fait toutefois en concordance avec les prescriptions ministérielles en matière de compétences.

8.1.4 Lors d'élaboration de nouveaux programmes, on se souciera de la protection de l'environnement et du développement durable. Du côté des DEC, il faut s'assurer, comme dans le paragraphe précédent, de la concordance avec les prescriptions ministérielles en matière de compétences. Pour ce qui est des AEC, il est possible de formuler ses propres compétences.

\subsection{Sensibilisation}

8.2.1 À chaque année, soutenir un club d'étudiantes et d'étudiants dont l'objectif serait de contribuer à la protection de l'environnement et au développement durable.

8.2.2 Réaliser à chaque année quatre activités de sensibilisation dont au moins une de masse destinée principalement à la clientèle étudiante où il sera question de protection de l'environnement et de développement durable.

8.2.3 Recevoir quatre (cégep vert - niveau 1) ou cinq formations (cégep vert - niveau 2 et suivants) en ÈRE à chaque année.

8.2.4 Déterminer une date à partir de laquelle les membres du personnel devront entre eux transmettre leurs communications écrites (mémo, lettre, document, etc.) de façon électronique.

8.2.5 Déterminer une date à partir de laquelle les enseignants et les enseignantes ainsi que les membres du personnel concernés devront communiquer avec leurs étudiantes et étudiants prioritairement de façon électronique.

8.2.6 À chaque fois que l'occasion s'y prête, faire part du souci de protection de l'environnement qu'a le Cégep dans ses communications tant à l'interne qu'à l'externe. 


\subsection{Gestion}

D'ici la fin de l'année scolaire 2008-2009, s'assurer que les principes de réduction, de réutilisation, de recyclage et de valorisation dans l'ensemble des activités du cégep soient respectés. Plus particulièrement en ce qui concerne :

\subsubsection{L'économie d'énergie}

En ce qui concerne les normes d'isolation, l'étanchéité des enveloppes thermiques et des fenêtres, les sources de chauffage et leur entretien, l'éclairage et la consommation d'énergie.

\subsubsection{La qualité de l'air}

En ce qui concerne l'entretien des conduits et des appareils de ventilation ainsi que l'analyse proprement dite de l'air.

\subsubsection{La gestion de l'eau}

En ce qui concerne l'état des conduites, le gaspillage, les dispositifs d'économie, la consommation des services, la qualité proprement dite de l'eau et son accessibilité et le traitement des eaux usées.

\subsubsection{La gestion des déchets}

En ce qui concerne le papier et le carton, l'acquisition de matériel provenant de matières recyclées ou pouvant être recyclables, la quantité et le type de déchets destinés au site d'enfouissement ainsi que ceux générés lors d'activités pratiquées en plein air.

\subsubsection{La gestion des matières dangereuses}

En ce qui concerne l'acquisition du volume strictement nécessaire, l'entreposage, les normes prescrites, les compétences requises pour manipuler, gérer et disposer de ces matières dangereuses, les protocoles concernant leur utilisation et les produits de remplacement plus respectueux de l'environnement.

\subsubsection{L'approvisionnement}

En ce qui concerne la politique d'approvisionnement à adapter à la politique environnementale, l'information à transmettre à nos fournisseurs et le choix éventuel de ceux-ci.

\subsubsection{L'entretien ménager}

En ce qui concerne l'utilisation de produits dédiés à l'entretien ménager de l'ensemble des espaces intérieurs des propriétés.

\subsubsection{L'aménagement extérieur}

En ce qui concerne l'utilisation de produits pour l'aménagement et l'entretien des espaces verts de même que pour l'entretien des aires de circulation.

\section{ARTICLE 9 - MODALITÉS D'APPLICATION}

À chaque année, le Cégep passe en revue les actions où la politique environnementale a dû s'appliquer. De la sorte, il évalue quels ont été les contraintes et les bénéfices afin de proposer des améliorations s'il y a lieu. Aussi, il s'assure de maintenir à jour ses connaissances en matière de développement des préoccupations environnementales au Québec ou ailleurs afin de demeurer à la fine pointe dans le domaine.

\section{ARTICLE 10 - ENTRÉE EN VIGUEUR}

La présente politique entre en vigueur dès qu'elle est adoptée par le conseil d'administration.

\section{ARTICLE 11 - RÉVISION}

La présente politique est révisée à chaque année pour les trois premières années et ensuite elle l'est aux trois ans.

Adoptée au conseil d'administration le 26 février 2007.

Modifiée le 28 avril 2008. 